\title{
Correction to: Thermoelastic response of non-simple solid cylinder with variable properties under a continuous line heat source
}

\author{
DS Mashat $^{1} \cdot$ AM Zenkour $^{1,2}$ (D) $\cdot$ AE Abouelregal ${ }^{3,4}$
}

Accepted: 22 October 2021 / Published online: 13 November 2021

(c) The Brazilian Society of Mechanical Sciences and Engineering 2021

\section{Correction to: Journal of the Brazilian Society of Mechanical Sciences and Engineering (2021) 43:240 https://doi.org/10.1007/s40430-021-02966-x}

In the original publication of the article, the Grant number mentioned in the acknowledgements section was published incorrectly.

The corrected version is given below.

The original article has been corrected.

Acknowledgements This project was funded by the Deanship of Scientific Research (DSR) at King Abdulaziz University, Jeddah, under Grant No. (G: 528-130-1441). The authors, therefore, acknowledge with thanks DSR for technical and financial support.
Publisher's Note Springer Nature remains neutral with regard to jurisdictional claims in published maps and institutional affiliations.

The original article can be found online at https://doi.org/10.1007/ s40430-021-02966-x.

AM Zenkour zenkour@kau.edu.sa

1 Department of Mathematics, Faculty of Science, King Abdulaziz University, Jeddah 21589, Saudi Arabia

2 Department of Mathematics, Faculty of Science, Kafrelsheikh University, Kafrelsheikh 33516, Egypt

3 Department of Mathematics, College of Science and Arts, Jouf University, Al-Qurayyat 75911, Saudi Arabia

4 Department of Mathematics, Faculty of Science, Mansoura University, Mansoura 35516, Egypt 